\begin{tabular}{l|l|c|} 
CRITICAL & $\begin{array}{l}\text { WCOSYSTEM } \\
\text { PARTNERSHIP FUND }\end{array}$ \\
\cline { 2 - 2 } & Special Series \\
\hline Meghamalai special section
\end{tabular}

\title{
BIRDS OF MEGHAMALAI LANDSCAPE, SOUTHERN WESTERN GHATS, INDIA
}

ISSN

Online 0974-7907 Print 0974-7893

\section{OPEN ACCESS}

\section{Santhanakrishnan Babu ${ }^{1} \&$ Subramanian Bhupathy ${ }^{2}$}

${ }^{1}$ Wildlife Information Liaison Development Society, 96 Kumudham Nagar, Vilankurichi Road, Coimbatore, Tamil Nadu 641035, India

${ }^{1,2}$ Sálim Ali Centre for Ornithology and Natural History, Anaikatti (PO), Coimbatore, Tamil Nadu 641108, India

${ }^{1}$ sanbabs@gmail.com (corresponding author), ${ }^{2}$ bhupathy.s@gmail.com

Abstract: Species composition of birds in the Meghamalai landscape with respect to threat status, foraging guild and biome-restricted assemblage were assessed based on data collected opportunistically during two research projects: first one spanned 36 months (20062009) the other for 18 months (June 2011-December 2012) and from literature published during mid 1940s. A total of 254 species belonging to 55 families and 18 orders were recorded, which include $11 \%$ (18 of 159 species) of globally threatened birds reported from India, 88\% (14 of 16 species) of endemic birds of the Western Ghats and a higher proportion of biome-restricted species (56\% of Indo-Malayan tropical dry zone and $80 \%$ of Indian Peninsula inhabited by tropical moist forest birds). Among the foraging guilds, insectivorous birds (51\%) dominated the bird composition followed by frugivores and carnivores. The present data shows that Meghamalai deserves to be recognized as an Important Bird Area of International Bird Conservation Network. This would enhance the conservation prospects of the landscape in a long run. The present study also highlights the importance of the area for conserving the birds of the Western Ghats.

Keywords: Endemic birds, foraging guild, High Wavy Mountains, IBA, Theni forests.
Around 1340 bird species have hitherto been reported from India (Manakadan \& Pittie 2001). As per IUCN (2012), 159 (12\%) bird species of the country are globally threatened (16 Critically Endangered, 17 Endangered, 54 Vulnerable, 69 Near Threatened and three Data Deficient), and they are distributed in a wide range of eco-regions (Ali \& Ripley 1983). The Western Ghats is one of the important eco-regions of India (Myers et al. 2000). It is a continuous mountain range ( 1600 $\mathrm{km}$ in length and $1,60,000 \mathrm{~km}^{2}$ in area) along the western side of peninsular India, across six states, viz., Kerala, Tamil Nadu, Karnataka, Goa, Maharashtra and Gujarat. A large extent of this mountain stretch has been altered for raising commercial plantations such as tea, coffee, cardamom and orchids (Nair 1999). Wide variation

DOI: http://dx.doi.org/10.11609/JoTT.03594.4962-72 | ZooBank: urn:Isid:zoobank.org:pub:F0A320C2-FD20-4D04-B976-6A753DE4CCF7

Editor: V. Santharam, Institute of Bird Studies \& Natural History, Chittoor, India.

Date of publication: 26 November 2013 (online \& print)

Manuscript details: Ms \# 03594 | Received 26 April 2013 | Final received 31 October 2013 | Finally accepted 04 November 2013

Citation: Babu, S. \& S. Bhupathy (2013). Birds of Meghamalai Landscape, southern Western Ghats, India. Journal of Threatened Taxa 5(15): 4962-4972; http:// dx.doi.org/10.11609/JoTT.03594.4962-72

Copyright: @ Babu \& Bhupathy 2013. Creative Commons Attribution 3.0 Unported License. JoTT allows unrestricted use of this article in any medium, reproduction and distribution by providing adequate credit to the authors and the source of publication.

Funding: ATREE-CEPF, Rufford Small Grant and Ministry of Environment and Forests, Government of India.

Competing Interest: Authors declare no competing interests.

Acknowledgements: We thank Tamil Nadu Forest Department for research permission. Our sincere thanks are due to Dr. P.A. Azeez and other colleagues at SACON and WILD for the facilities and support. Help rendered by WAR and VANAM during the surveys is highly appreciated.
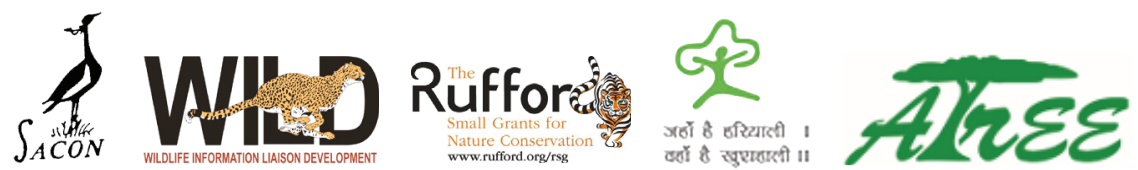

This article forms part of a special series on the Western Ghats of India, disseminating the results of work supported by the Critical Ecosystem Partnership Fund (CEPF), a joint initiative of l'Agence Française de Développement, Conservation International, the European Commission, the Global Environment Facility, the Government of Japan, the MacArthur Foundation and the World Bank. A fundamental goal of CEPF is to ensure civil society is engaged in biodiversity conservation. Implementation of the CEPF investment program in the Western Ghats is led and coordinated by the Ashoka Trust for Research in Ecology and the Environment (ATREE). 
in elevation (up to $2695 \mathrm{~m}$ in Anaimudi of Eravikulam National Park) and intensities of annual rainfall and pattern (rain-shadow and rain-fed found in the eastern and western side of plateau respectively) form diverse floral (dry thorn forests to tropical montane shola and grasslands) and faunal assemblages. The Western Ghats has been recognized as one of the eight hottest hotspots of global biodiversity for having a high degree of endemic animals $/ \mathrm{km}^{2}$ (Myers et al. 2000). Recently, 39 locations of the Ghats have been declared as World Heritage Sites by the UNESCO as an appreciation of their diverse and unique flora and fauna (Downloaded from http://whc.unesco.org/en/list/1342/ on 23 July 2013). Daniels (2003) reported 508 bird species from the Western Ghats including 16 endemics, and BirdLife International has identified the Western Ghats as an Endemic Bird Area (Statersfield 1998). Rasmussen \& Anderton (2005) reported 26 endemic species from the Western Ghats, but this is currently being debated. In the present paper, we consider 16 species as endemic to the Western Ghats.

Critical Ecosystem Partnership Fund (CEPF) program has accredited the Western Ghats and site-specific locations therein as high priority sites for protecting highly threatened ecosystems (CEPF 2007). One such site-specific location is Meghamalai. In a conservation perspective, the landscape acts as an important corridor linking Periyar Tiger Reserve and Grizzled Squirrel Wildlife Sanctuary.

Nichols (1944a,b; 1945) reported the occurrence of the birds of erstwhile Madura District including Meghamalai. After a lapse of over six decades, Bhupathy et al. (2012) collated some information on the birds of Meghamalai area. Subsequently, the first author while carrying out a CEPF-ATREE and Rufford Small Grant project systematically recorded birds of the area during June 2011-December 2012. The present study highlights the importance of the area for conserving birds in the Western Ghats.

\section{Materials and Methods}

The Meghamalai landscape $\left(9^{0} 31^{\prime}-9^{0} 51^{\prime} \mathrm{N} \& 77^{\circ} 10\right.$ $77^{0} 30^{\prime} \mathrm{E} ; \sim 480 \mathrm{~km}^{2}$ ) comes under the administrative control of the Theni Forest Division of Tamil Nadu State (Fig. 1). The area has been included under the PeriyarAgastiyamalai Corridor by Critical Ecosystem Partnership Fund (CEPF 2007). The major vegetation types include montane shola and grasslands, tropical wet evergreen, moist deciduous, dry deciduous forests, riparian and dry thorn forests, and economic crops (tea, coffee, cardamom etc) and plantations (softwood and hard wood). A major portion of the landscape receives precipitation from

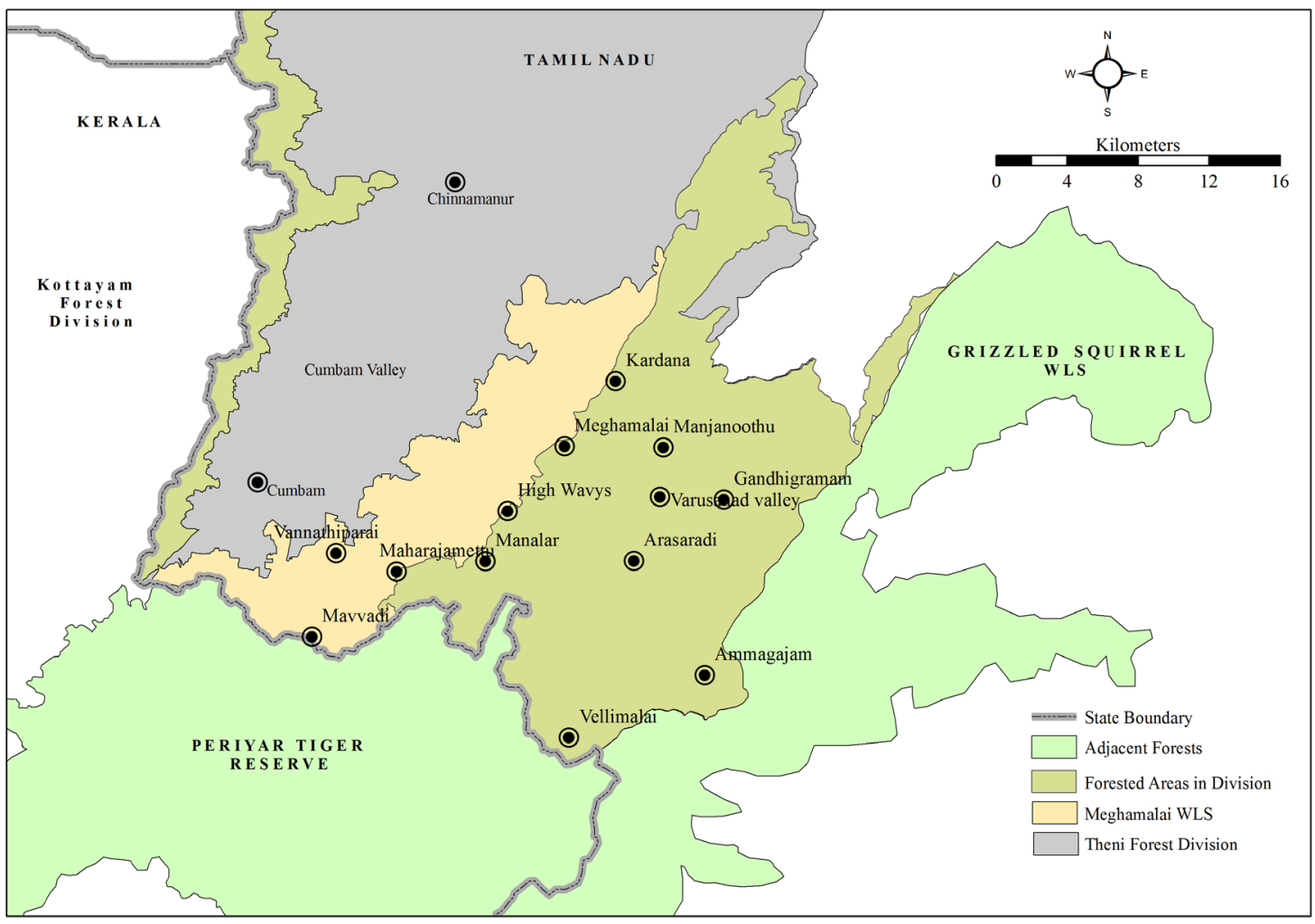

Figure 1. Surveyed areas in Meghamalai landscape, southern Western Ghats. 
the north-east monsoon, but the plateaus (hill tops) receive higher rainfall during the south-west monsoon. The northern slope of the landscape has recently been declared as "Meghamalai Wildlife Sanctuary" (Tamil Nadu Government Gazette (2009).

The occurrence of birds in the area was opportunistically recorded during field work by Sálim Ali Centre for Ornithology Natural History (2006-2009) and Wildlife Information Liaison Development Society (2011-2012). In addition, we also collated secondary data from published works (Nichols 1944a,b, 1945; Biddulph 1956). The nomenclature and details on distribution status (resident, resident migrant, migrants), endemism and feeding guild (aquatic vegetarian, granivore, frugivore, piscivore, carnivore, omnivore and nectarivore) were gleaned from Ali \& Ripley (1983). Threat status of each species observed/reported from the landscape was extracted from IUCN Red List (IUCN 2012). Biome-restricted assemblages of birds were categorized following (Islam \& Rahmani 2004).

\section{Results}

In total, 254 bird species belonging to 55 families and 18 orders were recorded in Meghamalai (Table 1), which consist of 10 migratory, 53 resident migratory and 191 resident species. This works out to be $19 \%$ of the bird fauna of India. Fourteen of them are endemic to the Western Ghats. Considerable populations of the Greybreasted Laughingthrush Garrulax jerdoni (6 individuals/ km in tea estates), Malabar Grey Hornbill Ocyceros griseus (flocks with over 30 individuals) and White-bellied Shortwing Brachypteryx major (0.8 individuals $/ \mathrm{km}$ in tea estates) were found. Out of 55 families, Muscicapidae (47 species) had the highest representation of species followed by Accipitridae (19) and Columbidae (12).

In all, 199 species of birds were added during recent studies and 23 recorded only in the past (1944-45). The Red-faced Malkoha Phaenicophaeus pyrrhocephalus could not be located during recent studies. Around 50\% and $19 \%$ of species reported from the Western Ghats and India were recorded from Meghamalai respectively. Coraciiformes recorded from Meghamalai accounted for $46 \%$ of species reported to occur in India followed by Cuculiformes (42\%) and Ciconiiformes (40\%).

All (14 species) endemics excluding Small Sunbird Nectarinia minima, Blue-winged Parakeet Psittacula columboides, Malabar Grey Hornbill and Grey-headed Bulbul Pycnonotus priocephalus were observed in upper reaches $(<1000 \mathrm{~m})$ forests. White-bellied Treepie Dendrocitta leucogastra was commonly observed in the coffee estates of Vellimalai and northwestern sides of
Varusanad Hills. Black-and-Orange Flycatcher Ficedula nigrorufa was recorded infrequently in Vellimalai and Manalar estates. Grey-breasted Laughingthrush was one of the most common species in the high elevations and a notable number were seen in Meghamalai Mountains, which starts from Gardana Estate in the east to Upper Manalar in the west. Nilgiri Pipit Anthus nilghiriensis and Broad-tailed Grass-Warbler Schoenicola platyura were seldom observed in grasslands between Maharaja Mettu and Mavadi Estate. Nilgiri Wood-Pigeon Columba elphinstonii was observed in the upper elevation (i.e., $>1000 \mathrm{~m}$ ) wet evergreen tracts of Upper Manalar and Vellimalai Estates.

Forty (15.7\%) species of birds recorded in the landscape were wetland associated while 214 species were forest dependent. Insectivores dominated the bird assemblage (130 species, 51\%) followed by frugivores (34 species, 13\%) and carnivores (28 species, 11\%). Aquatic (herbivores) and nectarivores contributed the least (each 5 species, Fig. 2).

In all, 18 globally threatened birds were recorded from this landscape and included the Critically Endangered Long-billed Vulture Gyps indicus; and White-bellied Shortwing; Vulnerable Broad-tailed Grass-Warbler Schoenicola platyura, Yellow-throated Bulbul Pycnonotus xantholaemus, Nilgiri Pipit Anthus nilghiriensis and Nilgiri Wood-pigeon and 11 Near Threatened species (Fig. 3). Out of the 11 Near Threatened, four were aquatic while the other seven were terrestrial species. Among the threatened species of the area, Long-billed Vulture and Grey-headed Fish-Eagle (Lesser/Greater) were historical reports.

\section{Discussion}

Species richness of birds and number of endemics recorded in Meghamalai landscape is comparable to

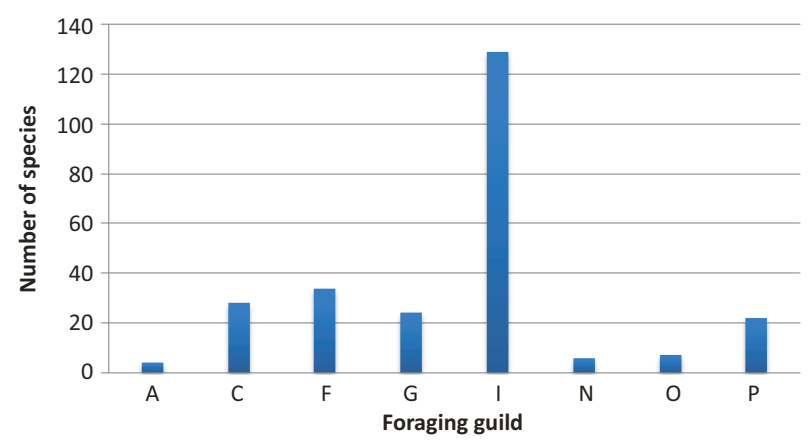

Figure 2. Composition of birds belonging to various foraging guilds in Meghamalai landscape.

I - Insectivores, N - Nectarivores, O - Omnivores, P - Piscivores,

A - Aquatic (herbivores), C - Carnivores, F - Frugivores, G - Granivores 


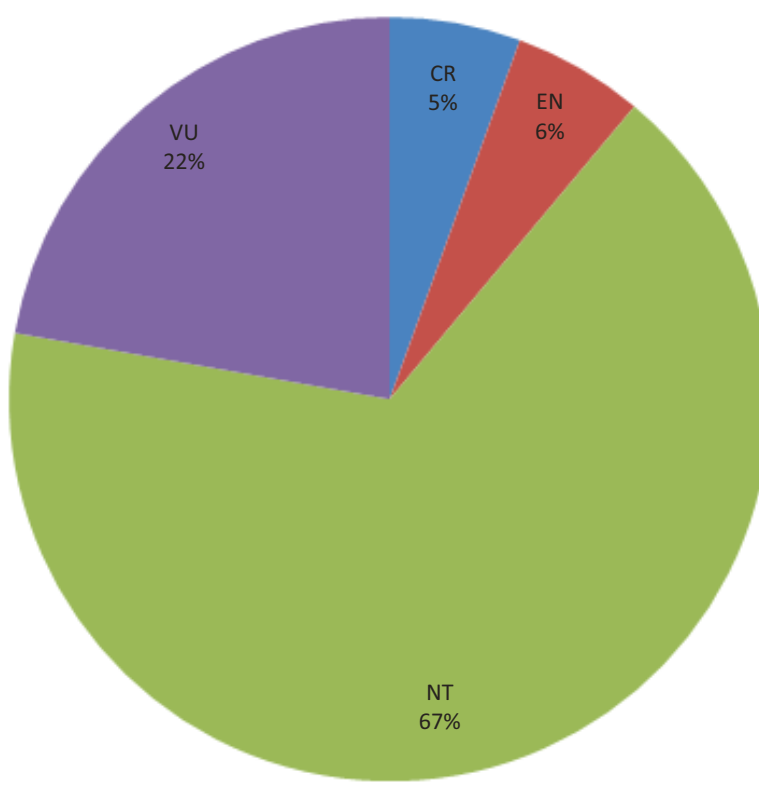

Figure 3. Composition of threatened birds in Meghamalai as per IUCN criteria.

NT - Near Threatened, VU - Vulnerable, EN - Endangered, $\mathrm{CR}$ - Critically Endangered

other forest areas located largely on the leeward side of the southern Western Ghats such as the Mudumalai Wildlife Sanctuary (266 species, nine endemics; Gokula \& Vijayan 1996), Palni Hills (277 species, 13 endemics; Balachandran et al. 2005), Anamalai Tiger Reserve (218 species, 12 endemics; Kannan 1998), KalakkadMundanthurai Tiger Reserve (273 species 15 endemics; Johnsingh 2001) and Grizzled Squirrel Wildlife Sanctuary (220 species, 14 endemics; Islam \& Rahmani 2004). Vegetation composition, altitudinal gradient of the above areas, and sampling efforts in previous studies were different compared to the present study. The record of 254 species in the landscape is around $50 \%$ of 508 recorded birds of the Western Ghats. It is to be noted that the present bird list of Meghamalai is incomplete and further intensive studies would add more species.

High species richness of birds in the landscape could be attributed to terrain (wide altitudinal gradient: 200$2000 \mathrm{~m}$ ), rainfall pattern (leeward and windward zones) and vegetation characteristics (highly mosaic habitats under different disturbance levels). Further, diverse order and family representations of birds in the area indicate the availability of diverse and abundant food resources, which is an important factor determining the community structure (Recher \& Davis 2002).

One vulture species (Long-billed Vulture Gyps indicus) has been reported during the 1940s from the erstwhile Madura District (Nichols 1944a,b, 1945), however recent surveys were unsuccessful in locating the same. The dramatic decline of Gyps vultures in the Indian subcontinent has been reported by Prakash et al. (2012). Twenty species of birds reported by Nichols (1944a,b, 1945;) were not recorded all in recent surveys (20062012; Table 1). This could be due to the disappearance of species such as vultures from the area. Furthermore, one carnivorous, four granivorous, 12 insectivorous and three piscivorous birds reported earlier were also not recorded during recent studies (Table 1). Endemic species, Grey-breasted Laughing-thrush, Nilgiri-Wood Pigeon, Nilgiri Pipit and Nilgiri Flycatcher, recorded during the present studies were not reported earlier. Incomplete spatial and temporal coverage in these surveys and habitat changes due to plantations, and other anthropogenic activities over the years might have caused these variations. This indicates our incomplete understanding on the bird fauna of Meghamalai. The only sight record of Red-faced Malkoha Phaenicophaeus pyrrhocephalus is reported from low elevation forests of Meghamalai (Biddulph 1956). However, Hoffmann (1996) reported this as a spurious record. Furthermore, this species was not observed during the recent studies.

The high proportion of endemics in this area is accredited to the large extent of high elevation ( $<1400 \mathrm{~m})$ forests, south of Anamalais (see Bhupathy \& Babu 2013). High elevation forests are reported to harbor relatively larger number of endemics in the Western Ghats. Furthermore contiguous patches of evergreen forests still persist on the western side of the landscape, where it is merges with Periyar Tiger Reserve. With respect to feeding guilds, insectivores dominated the bird composition of Meghamalai followed by frugivore and carnivores. A similar pattern of guild structure was observed in Mudumalai Wildlife Sanctuary (Gokula \& Vijayan 1996), Palni Hills (Somasundaram \& Vijayan 2008), Mundanthurai plateau (Johnsingh \& Joshua 1994) and Anaikatty (Nirmala 2002) of Western Ghats. Representation of major foraging guilds in the area indicated that the area holds wide food resources.

The high degree of globally threatened (18 species $=11 \%$ of India's threatened birds), range restricted (14 species $=88 \%$ of Western Ghats endemics) and biomerestricted species (i.e., 33 species (56\%) of 59 species of Indo-Malayan tropical dry zone and 12 of 15 species (80\%) of Indian peninsula tropical moist forest) in Meghamalai fulfill criteria set for recognizing a location as an Important Bird Area (IBA; Islam \& Rahmani 2004). Recognition of Meghamalai as one of the IBAs of the country would pave the way for further conservation initiatives in the landscape for wildlife, especially birds. 
Table 1. Checklist of birds recorded in Meghamalai landscape, southern Western Ghats, India. Names after Manakadan \& Pitte (2001).

\begin{tabular}{|c|c|c|c|c|c|}
\hline $\begin{array}{l}\text { Family / Common } \\
\text { Name }\end{array}$ & Scientific Name & IUCN & Status & Guild & Source \\
\hline \multicolumn{6}{|l|}{ Podicipedidae } \\
\hline Little Grebe & $\begin{array}{l}\text { Tachybaptus } \\
\text { ruficollis }\end{array}$ & LC & $\mathrm{R}$ & $P$ & 2,3 \\
\hline \multicolumn{6}{|l|}{ Pelecanidae } \\
\hline Spot-billed Pelican & $\begin{array}{l}\text { Pelecanus } \\
\text { philippensis }\end{array}$ & NT & RM & $P$ & 3 \\
\hline \multicolumn{6}{|l|}{ Phalacrocoracidae } \\
\hline Little Cormorant & $\begin{array}{l}\text { Phalacrocorax } \\
\text { niger }\end{array}$ & LC & RM & $P$ & $1,2,3$ \\
\hline Indian Shag & $\begin{array}{l}\text { Phalacrocorax } \\
\text { fuscicollis }\end{array}$ & LC & RM & $P$ & 3 \\
\hline \multicolumn{6}{|l|}{ Anhingidae } \\
\hline Darter & $\begin{array}{l}\text { Anhinga } \\
\text { melanogaster }\end{array}$ & NT & RM & $P$ & 3 \\
\hline \multicolumn{6}{|l|}{ Ardeidae } \\
\hline Purple Heron & Ardea purpurea & LC & RM & $P$ & 1,3 \\
\hline Grey Heron & Ardea cinerea & LC & RM & $P$ & 3 \\
\hline Large Egret & Casmerodius albus & LC & RM & $P$ & 3 \\
\hline Cattle Egret & Bubulcus ibis & LC & RM & $\mathrm{P}$ & 2,3 \\
\hline Yellow Bittern & Ixobrychus sinensis & LC & RM & $P$ & 1 \\
\hline Chestnut Bittern & $\begin{array}{l}\text { Ixobrychus } \\
\text { cinnamomeus }\end{array}$ & LC & RM & $P$ & 1 \\
\hline Little Egret & Egretta garzetta & LC & $R$ & $P$ & 2,3 \\
\hline Indian Pond-Heron & Ardeola grayii & LC & $\mathrm{R}$ & $P$ & 2,3 \\
\hline $\begin{array}{l}\text { Black-crowned } \\
\text { Night-Heron }\end{array}$ & $\begin{array}{l}\text { Nycticorax } \\
\text { nycticorax }\end{array}$ & LC & $\mathrm{R}$ & $P$ & 1,2 \\
\hline \multicolumn{6}{|l|}{ Ciconiidae } \\
\hline Painted Stork & $\begin{array}{l}\text { Mycteria } \\
\text { leucocephala }\end{array}$ & NT & RM & $P$ & 3 \\
\hline $\begin{array}{l}\text { Asian Openbill- } \\
\text { Stork }\end{array}$ & Anastomus oscitans & LC & $\mathrm{R}$ & 1 & 3 \\
\hline $\begin{array}{l}\text { White-necked } \\
\text { Stork }\end{array}$ & Ciconia episcopus & LC & $R$ & $\mathrm{P}$ & 3 \\
\hline \multicolumn{6}{|l|}{ Threskiornithidae } \\
\hline Oriental White Ibis & $\begin{array}{l}\text { Threskiornis } \\
\text { melanocephalus }\end{array}$ & NT & $R$ & 1 & 3 \\
\hline Black Ibis & Pseudibis papillosa & LC & R & 1 & 3 \\
\hline \multicolumn{6}{|l|}{ Anatidae } \\
\hline $\begin{array}{l}\text { Lesser Whistling- } \\
\text { Duck }\end{array}$ & $\begin{array}{l}\text { Dendrocygna } \\
\text { javanica }\end{array}$ & LC & $R$ & A & 3 \\
\hline Spot-billed Duck & $\begin{array}{l}\text { Anas } \\
\text { poecilorhyncha }\end{array}$ & LC & RM & $A$ & 3 \\
\hline \multicolumn{6}{|l|}{ Accipitridae } \\
\hline Black Baza & Aviceda leuphotes & LC & RM & C & 3 \\
\hline $\begin{array}{l}\text { Oriental Honey- } \\
\text { Buzzard }\end{array}$ & $\begin{array}{l}\text { Pernis } \\
\text { ptilorhynchus }\end{array}$ & LC & RM & C & $1,2,3$ \\
\hline Pallid Harrier & Circus macrourus & NT & M & c & 2 \\
\hline Montagu's Harrier & Circus pygargus & LC & $M$ & c & 2 \\
\hline $\begin{array}{l}\text { Eurasian } \\
\text { Sparrowhawk }\end{array}$ & Accipiter nisus & LC & RM & C & 2 \\
\hline
\end{tabular}

\begin{tabular}{|c|c|c|c|c|c|}
\hline $\begin{array}{l}\text { Family / Common } \\
\text { Name }\end{array}$ & Scientific Name & IUCN & Status & Guild & Source \\
\hline Jerdon's Baza & Aviceda jerdoni & LC & $\mathrm{R}$ & C & 2 \\
\hline $\begin{array}{l}\text { Black-shouldered } \\
\text { Kite }\end{array}$ & Elanus caeruleus & LC & $\mathrm{R}$ & C & 2,3 \\
\hline Black Kite & Milvus migrans & LC & $R$ & 0 & 2,3 \\
\hline Brahminy Kite & Haliastur indus & LC & $\mathrm{R}$ & 0 & 2,3 \\
\hline $\begin{array}{l}\text { Grey-headed } \\
\text { Fish-Eagle (Lesser/ } \\
\text { Greater) }\end{array}$ & $\begin{array}{l}\text { Ichthyophaga } \\
\text { ichthyaetus /I. } \\
\text { humilis }\end{array}$ & NT & $\mathrm{R}$ & $P$ & 1 \\
\hline $\begin{array}{l}\text { Short-toed Snake- } \\
\text { Eagle }\end{array}$ & Circaetus gallicus & LC & $\mathrm{R}$ & C & 3 \\
\hline Long-billed Vulture & Gyps indicus & $\mathrm{CR}$ & $R$ & C & 1 \\
\hline $\begin{array}{l}\text { Crested Serpent- } \\
\text { Eagle }\end{array}$ & Spilornis cheela & LC & $\mathrm{R}$ & C & 2,3 \\
\hline Black Eagle & $\begin{array}{l}\text { Ictinaetus } \\
\text { malayensis }\end{array}$ & LC & $R$ & C & 4 \\
\hline Booted Eagle & $\begin{array}{l}\text { Hieraaetus } \\
\text { pennatus }\end{array}$ & LC & RM & C & 4 \\
\hline Shikra & Accipiter badius & LC & $R$ & C & $1,2,3$ \\
\hline $\begin{array}{l}\text { Changeable Hawk- } \\
\text { Eagle }\end{array}$ & Spizaetus cirrhatus & LC & $\mathrm{R}$ & C & 2 \\
\hline $\begin{array}{l}\text { Rufous-bellied } \\
\text { Eagle }\end{array}$ & Hieraaetus kienerii & LC & $\mathrm{R}$ & C & 1 \\
\hline \multicolumn{6}{|l|}{ Falconidae } \\
\hline Common Kestrel & Falco tinnunculus & LC & RM & C & 2,3 \\
\hline Red-headed Falcon & Falco chicquera & LC & $R$ & C & 2 \\
\hline \multicolumn{6}{|l|}{ Phasianidae } \\
\hline Rain Quail & $\begin{array}{l}\text { Coturnix } \\
\text { coromandelica }\end{array}$ & LC & RM & G & 2 \\
\hline Grey Francolin & $\begin{array}{l}\text { Francolinus } \\
\text { pondicerianus }\end{array}$ & LC & $R$ & G & 3 \\
\hline Jungle Bush-Quail & Perdicula asiatica & LC & $R$ & G & 3 \\
\hline $\begin{array}{l}\text { Blue-breasted } \\
\text { Quail }\end{array}$ & Coturnix chinensis & LC & $\mathrm{R}$ & G & 1 \\
\hline Red Spurfowl & $\begin{array}{l}\text { Galloperdix } \\
\text { spadicea }\end{array}$ & LC & $\mathrm{R}$ & G & 2,3 \\
\hline Grey Junglefowl & Gallus sonneratii & LC & $\mathrm{R}$ & G & 2,3 \\
\hline Indian Peafowl & Pavo cristatus & LC & $\mathrm{R}$ & G & 2,3 \\
\hline \multicolumn{6}{|l|}{ Turnicidae } \\
\hline $\begin{array}{l}\text { Common } \\
\text { Buttonquail }\end{array}$ & Turnix suscitator & LC & $R$ & G & 1 \\
\hline \multicolumn{6}{|l|}{ Rallidae } \\
\hline Slaty-legged Crake & $\begin{array}{l}\text { Rallina } \\
\text { eurizonoides }\end{array}$ & LC & RM & I & 1 \\
\hline $\begin{array}{l}\text { Ruddy-breasted } \\
\text { Crake }\end{array}$ & Porzana fusca & LC & $R$ & I & 1 \\
\hline $\begin{array}{l}\text { White-breasted } \\
\text { Waterhen }\end{array}$ & $\begin{array}{l}\text { Amaurornis } \\
\text { phoenicurus }\end{array}$ & LC & $R$ & 1 & 3 \\
\hline Purple Moorhen & Porphyrio porphyrio & LC & $R$ & A & 3 \\
\hline \multicolumn{6}{|l|}{ Jacanidae } \\
\hline $\begin{array}{l}\text { Pheasant-tailed } \\
\text { Jacana }\end{array}$ & $\begin{array}{l}\text { Hydrophasianus } \\
\text { chirurgus }\end{array}$ & LC & $\mathrm{R}$ & A & 3 \\
\hline
\end{tabular}




\begin{tabular}{|c|c|c|c|c|c|}
\hline $\begin{array}{l}\text { Family / Common } \\
\text { Name }\end{array}$ & Scientific Name & IUCN & Status & Guild & Source \\
\hline \multicolumn{6}{|l|}{ Rostratulidae } \\
\hline $\begin{array}{l}\text { Greater Painted- } \\
\text { Snipe }\end{array}$ & $\begin{array}{l}\text { Rostratula } \\
\text { benghalensis }\end{array}$ & LC & $\mathrm{R}$ & G & 1 \\
\hline \multicolumn{6}{|l|}{ Charadriidae } \\
\hline $\begin{array}{l}\text { Yellow-wattled } \\
\text { Lapwing }\end{array}$ & $\begin{array}{l}\text { Vanellus } \\
\text { malabaricus }\end{array}$ & LC & $\mathrm{R}$ & 1 & 3 \\
\hline $\begin{array}{l}\text { Red-wattled } \\
\text { Lapwing }\end{array}$ & Vanellus indicus & LC & $\mathrm{R}$ & I & 2,3 \\
\hline \multicolumn{6}{|l|}{ Scolopacidae } \\
\hline $\begin{array}{l}\text { Common } \\
\text { Sandpiper }\end{array}$ & Actitis hypoleucos & LC & RM & 1 & 1,3 \\
\hline \multicolumn{6}{|l|}{ Pteroclididae } \\
\hline $\begin{array}{l}\text { Chestnut-bellied } \\
\text { Sandgrouse }\end{array}$ & Pterocles exustus & LC & $\mathrm{R}$ & G & 1 \\
\hline \multicolumn{6}{|l|}{ Columbidae } \\
\hline Blue Rock Pigeon & Columba livia & LC & $R$ & G & 2,3 \\
\hline $\begin{array}{l}\text { Nilgiri Wood- } \\
\text { Pigeon }\end{array}$ & $\begin{array}{l}\text { Columba } \\
\text { elphinstonii * }\end{array}$ & VU & $\mathrm{R}$ & $\mathrm{F}$ & 3 \\
\hline $\begin{array}{l}\text { Oriental Turtle } \\
\text { Dove }\end{array}$ & $\begin{array}{l}\text { Streptopelia } \\
\text { orientalis }\end{array}$ & LC & RM & G & 4 \\
\hline Little Brown Dove & $\begin{array}{l}\text { Streptopelia } \\
\text { senegalensis }\end{array}$ & LC & $\mathrm{R}$ & G & 2,3 \\
\hline Spotted Dove & $\begin{array}{l}\text { Streptopelia } \\
\text { chinensis }\end{array}$ & LC & $\mathrm{R}$ & G & 2,3 \\
\hline $\begin{array}{l}\text { Eurasian Collared- } \\
\text { Dove }\end{array}$ & $\begin{array}{l}\text { Streptopelia } \\
\text { decaocto }\end{array}$ & LC & $\mathrm{R}$ & G & 2,3 \\
\hline Emerald Dove & Chalcophaps indica & LC & $R$ & G & 2,3 \\
\hline $\begin{array}{l}\text { Orange-breasted } \\
\text { Green-Pigeon }\end{array}$ & Treron bicincta & LC & $\mathrm{R}$ & $\mathrm{F}$ & 3 \\
\hline $\begin{array}{l}\text { Pompadour Green- } \\
\text { Pigeon }\end{array}$ & Treron pompadora & LC & $\mathrm{R}$ & $\mathrm{F}$ & 1,3 \\
\hline $\begin{array}{l}\text { Yellow-legged } \\
\text { Green-Pigeon }\end{array}$ & $\begin{array}{l}\text { Treron } \\
\text { phoenicoptera }\end{array}$ & LC & $R$ & $\mathrm{~F}$ & 3 \\
\hline $\begin{array}{l}\text { Green Imperial- } \\
\text { Pigeon }\end{array}$ & Ducula aenea & LC & $\mathrm{R}$ & $\mathrm{F}$ & 1,3 \\
\hline $\begin{array}{l}\text { Mountain Imperial- } \\
\text { Pigeon }\end{array}$ & Ducula badia & LC & $\mathrm{R}$ & $\mathrm{F}$ & 3 \\
\hline \multicolumn{6}{|l|}{ Psittacidae } \\
\hline $\begin{array}{l}\text { Rose-ringed } \\
\text { Parakeet }\end{array}$ & Psittacula krameri & LC & $\mathrm{R}$ & $\mathrm{F}$ & 2,3 \\
\hline $\begin{array}{l}\text { Indian Hanging- } \\
\text { Parrot }\end{array}$ & Loriculus vernalis & LC & $\mathrm{R}$ & $\mathrm{F}$ & 2,3 \\
\hline $\begin{array}{l}\text { Plum-headed } \\
\text { Parakeet }\end{array}$ & $\begin{array}{l}\text { Psittacula } \\
\text { cyanocephala }\end{array}$ & LC & $\mathrm{R}$ & $\mathrm{F}$ & 2,3 \\
\hline $\begin{array}{l}\text { Blue-winged } \\
\text { Parakeet }\end{array}$ & $\begin{array}{l}\text { Psittacula } \\
\text { columboides * }\end{array}$ & LC & $\mathrm{R}$ & G & 2,3 \\
\hline \multicolumn{6}{|l|}{ Cuculidae } \\
\hline $\begin{array}{l}\text { Pied Crested } \\
\text { Cuckoo }\end{array}$ & Clamator jacobinus & LC & RM & I & 2,3 \\
\hline Common Cuckoo & Cuculus canorus & LC & RM & I & 3 \\
\hline $\begin{array}{l}\text { Banded Bay } \\
\text { Cuckoo }\end{array}$ & $\begin{array}{l}\text { Cacomantis } \\
\text { sonneratii }\end{array}$ & LC & $\mathrm{R}$ & I & 3 \\
\hline Asian Koel & $\begin{array}{l}\text { Eudynamys } \\
\text { scolopacea }\end{array}$ & LC & $\mathrm{R}$ & I & 2,3 \\
\hline Brainfever Bird & Hierococcyx varius & LC & $\mathrm{R}$ & I & 2,3 \\
\hline
\end{tabular}

\begin{tabular}{|c|c|c|c|c|c|}
\hline $\begin{array}{l}\text { Family / Common } \\
\text { Name }\end{array}$ & Scientific Name & IUCN & Status & Guild & Source \\
\hline $\begin{array}{l}\text { Indian Plaintive } \\
\text { Cuckoo }\end{array}$ & $\begin{array}{l}\text { Cacomantis } \\
\text { passerinus }\end{array}$ & LC & $\mathrm{R}$ & 1 & 3 \\
\hline Drongo Cuckoo & Surniculus lugubris & LC & $\mathrm{R}$ & 1 & 3 \\
\hline $\begin{array}{l}\text { Small Green-billed } \\
\text { Malkoha }\end{array}$ & $\begin{array}{l}\text { Phaenicophaeus } \\
\text { viridirostris }\end{array}$ & LC & $\mathrm{R}$ & 1 & 2,3 \\
\hline Sirkeer Malkoha & $\begin{array}{l}\text { Phaenicophaeus } \\
\text { leschenaultii }\end{array}$ & LC & $\mathrm{R}$ & 1 & 2,3 \\
\hline Greater Coucal & Centropus sinensis & LC & $\mathrm{R}$ & 1 & 2,3 \\
\hline \multicolumn{6}{|l|}{ Tytonidae } \\
\hline Barn Owl & Tyto alba & LC & $\mathrm{R}$ & C & 2,3 \\
\hline \multicolumn{6}{|l|}{ Strigidae } \\
\hline Forest Eagle-Owl & Bubo nipalensis & LC & $\mathrm{R}$ & C & $1,2,3$ \\
\hline Oriental Scops-Owl & Otus sunia & LC & $\mathrm{R}$ & C & 2,3 \\
\hline Eurasian Eagle-Owl & Bubo bubo & LC & $\mathrm{R}$ & C & 2,3 \\
\hline Collared Scops-Owl & Otus bakkamoena & LC & $\mathrm{R}$ & C & 2,3 \\
\hline Brown Fish-Owl & Ketupa zeylonensis & LC & $\mathrm{R}$ & C & 3 \\
\hline Mottled Wood-Owl & Strix ocellata & LC & $\mathrm{R}$ & C & 2,3 \\
\hline Brown Wood-Owl & $\begin{array}{l}\text { Strix } \\
\text { leptogrammica }\end{array}$ & LC & $\mathrm{R}$ & C & 1,3 \\
\hline Jungle Owlet & $\begin{array}{l}\text { Glaucidium } \\
\text { radiatum }\end{array}$ & LC & $\mathrm{R}$ & C & 2,3 \\
\hline Spotted Owlet & Athene brama & LC & $\mathrm{R}$ & C & 2,3 \\
\hline Brown Hawk-Owl & Ninox scutulata & LC & $\mathrm{R}$ & C & 1,3 \\
\hline \multicolumn{6}{|l|}{ Caprimulgidae } \\
\hline $\begin{array}{l}\text { Indian Jungle } \\
\text { Nightjar }\end{array}$ & $\begin{array}{l}\text { Caprimulgus } \\
\text { indicus }\end{array}$ & LC & RM & 1 & 2,3 \\
\hline Jerdon's Nightjar & $\begin{array}{l}\text { Caprimulgus } \\
\text { atripennis }\end{array}$ & LC & RM & I & 1 \\
\hline $\begin{array}{l}\text { Common Indian } \\
\text { Nightjar }\end{array}$ & $\begin{array}{l}\text { Caprimulgus } \\
\text { asiaticus }\end{array}$ & LC & $\mathrm{R}$ & 1 & 2,3 \\
\hline \multicolumn{6}{|l|}{ Apodidae } \\
\hline $\begin{array}{l}\text { Indian Edible-nest } \\
\text { Swiftlet }\end{array}$ & Collocalia unicolor & LC & $\mathrm{R}$ & 1 & 4 \\
\hline House Swift & Apus affinis & LC & RM & 1 & 3 \\
\hline Asian Palm-Swift & $\begin{array}{l}\text { Cypsiurus } \\
\text { balasiensis }\end{array}$ & LC & $\mathrm{R}$ & 1 & 3 \\
\hline \multicolumn{6}{|l|}{ Trogonidae } \\
\hline Malabar Trogon & Harpactes fasciatus & LC & $\mathrm{R}$ & I & 2,3 \\
\hline \multicolumn{6}{|l|}{ Alcedinidae } \\
\hline $\begin{array}{l}\text { Small Blue } \\
\text { Kingfisher }\end{array}$ & Alcedo atthis & LC & RM & $P$ & 2,3 \\
\hline $\begin{array}{l}\text { Black-capped } \\
\text { Kingfisher }\end{array}$ & Halcyon pileata & LC & $\mathrm{R}$ & $\mathrm{P}$ & 3 \\
\hline $\begin{array}{l}\text { White-breasted } \\
\text { Kingfisher }\end{array}$ & Halcyon smyrnensis & LC & $\mathrm{R}$ & $P$ & 2,3 \\
\hline $\begin{array}{l}\text { Stork-billed } \\
\text { Kingfisher }\end{array}$ & Halcyon capensis & LC & $\mathrm{R}$ & $P$ & 1 \\
\hline $\begin{array}{l}\text { Lesser Pied } \\
\text { Kingfisher }\end{array}$ & Ceryle rudis & LC & $\mathrm{R}$ & $P$ & 3 \\
\hline \multicolumn{6}{|l|}{ Meropidae } \\
\hline $\begin{array}{l}\text { Blue-tailed Bee- } \\
\text { eater }\end{array}$ & Merops philippinus & LC & RM & 1 & 1,2 \\
\hline
\end{tabular}




\begin{tabular}{|c|c|c|c|c|c|}
\hline $\begin{array}{l}\text { Family / Common } \\
\text { Name }\end{array}$ & Scientific Name & IUCN & Status & Guild & Source \\
\hline $\begin{array}{l}\text { Blue-bearded Bee- } \\
\text { eater }\end{array}$ & $\begin{array}{l}\text { Nyctyornis } \\
\text { athertoni }\end{array}$ & LC & $\mathrm{R}$ & 1 & 3 \\
\hline Small Bee-eater & Merops orientalis & LC & $R$ & I & 2,3 \\
\hline $\begin{array}{l}\text { Chestnut-headed } \\
\text { Bee-eater }\end{array}$ & $\begin{array}{l}\text { Merops } \\
\text { leschenaulti }\end{array}$ & LC & $\mathrm{R}$ & 1 & 2,3 \\
\hline \multicolumn{6}{|l|}{ Coraciidae } \\
\hline Indian Roller & $\begin{array}{l}\text { Coracias } \\
\text { benghalensis }\end{array}$ & LC & RM & 1 & 2,3 \\
\hline \multicolumn{6}{|l|}{ Upupidae } \\
\hline Common Hoopoe & Upupa epops & LC & RM & 1 & 2,3 \\
\hline \multicolumn{6}{|l|}{ Bucerotidae } \\
\hline $\begin{array}{l}\text { Malabar Pied } \\
\text { Hornbill }\end{array}$ & $\begin{array}{l}\text { Anthracoceros } \\
\text { coronatus }\end{array}$ & NT & $\mathrm{R}$ & $\mathrm{F}$ & 2 \\
\hline $\begin{array}{l}\text { Great Pied Hornbill } \\
\text { (Image 1) }\end{array}$ & Buceros bicornis & NT & $\mathrm{R}$ & $\mathrm{F}$ & $1,2,3$ \\
\hline $\begin{array}{l}\text { Indian Grey } \\
\text { Hornbill }\end{array}$ & Ocyceros birostris & LC & $\mathrm{R}$ & $\mathrm{F}$ & 2 \\
\hline $\begin{array}{l}\text { Malabar Grey } \\
\text { Hornbill }\end{array}$ & Ocyceros griseus * & LC & $\mathrm{R}$ & $\mathrm{F}$ & 2,3 \\
\hline \multicolumn{6}{|l|}{ Capitonidae } \\
\hline $\begin{array}{l}\text { White-cheeked } \\
\text { Barbet }\end{array}$ & Megalaima viridis & LC & $\mathrm{R}$ & $\mathrm{F}$ & $1,2,3$ \\
\hline $\begin{array}{l}\text { Brown-headed } \\
\text { Barbet }\end{array}$ & $\begin{array}{l}\text { Megalaima } \\
\text { zeylanica }\end{array}$ & LC & $\mathrm{R}$ & $\mathrm{F}$ & 2,3 \\
\hline $\begin{array}{l}\text { Crimson-throated } \\
\text { Barbet }\end{array}$ & $\begin{array}{l}\text { Megalaima } \\
\text { rubricapillus }\end{array}$ & LC & $\mathrm{R}$ & $\mathrm{F}$ & 2,3 \\
\hline $\begin{array}{l}\text { Coppersmith } \\
\text { Barbet }\end{array}$ & $\begin{array}{l}\text { Megalaima } \\
\text { haemacephala }\end{array}$ & LC & $\mathrm{R}$ & $\mathrm{F}$ & 2,3 \\
\hline \multicolumn{6}{|l|}{ Picidae } \\
\hline $\begin{array}{l}\text { Rufous } \\
\text { Woodpecker }\end{array}$ & Celeus brachyurus & LC & $\mathrm{R}$ & 1 & 1,2 \\
\hline $\begin{array}{l}\text { Brown- } \\
\text { capped Pygmy } \\
\text { Woodpecker }\end{array}$ & Dendrocopos nanus & LC & $\mathrm{R}$ & 1 & 3,4 \\
\hline $\begin{array}{l}\text { Yellow-fronted } \\
\text { Pied Woodpecker }\end{array}$ & $\begin{array}{l}\text { Dendrocopos } \\
\text { mahrattensis }\end{array}$ & LC & $\mathrm{R}$ & 1 & 4 \\
\hline $\begin{array}{l}\text { Little Scaly-bellied } \\
\text { Green Woodpecker }\end{array}$ & $\begin{array}{l}\text { Picus } \\
\text { xanthopygaeus }\end{array}$ & LC & $\mathrm{R}$ & 1 & 3 \\
\hline $\begin{array}{l}\text { Small Yellow-naped } \\
\text { Woodpecker }\end{array}$ & Picus chlorolophus & LC & $R$ & 1 & 1 \\
\hline $\begin{array}{l}\text { Common } \\
\text { Golden-backed } \\
\text { Woodpecker }\end{array}$ & $\begin{array}{l}\text { Dinopium } \\
\text { javanense }\end{array}$ & LC & $R$ & 1 & 3,4 \\
\hline $\begin{array}{l}\text { Lesser Golden- } \\
\text { backed } \\
\text { Woodpecker }\end{array}$ & $\begin{array}{l}\text { Dinopium } \\
\text { benghalense }\end{array}$ & LC & $\mathrm{R}$ & 1 & $1,2,3$ \\
\hline $\begin{array}{l}\text { Heart-spotted } \\
\text { Woodpecker }\end{array}$ & Hemicircus canente & LC & $R$ & I & 1,3 \\
\hline $\begin{array}{l}\text { Black-shouldered } \\
\text { Woodpecker }\end{array}$ & $\begin{array}{l}\text { Chrysocolaptes } \\
\text { festivus }\end{array}$ & LC & $\mathrm{R}$ & I & 1 \\
\hline \multicolumn{6}{|l|}{ Pittidae } \\
\hline Indian Pitta & Pitta brachyura & LC & $R$ & I & $1,2,3$ \\
\hline \multicolumn{6}{|l|}{ Alaudidae } \\
\hline $\begin{array}{l}\text { Ashy-crowned } \\
\text { Sparrow-Lark }\end{array}$ & Eremopterix grisea & LC & $R$ & 1 & 2 \\
\hline
\end{tabular}

\begin{tabular}{|c|c|c|c|c|c|}
\hline $\begin{array}{l}\text { Family / Common } \\
\text { Name }\end{array}$ & Scientific Name & IUCN & Status & Guild & Source \\
\hline Jerdon's Bush-Lark & Mirafra affinis & LC & $\mathrm{R}$ & 1 & 1 \\
\hline $\begin{array}{l}\text { Rufous-tailed } \\
\text { Finch-Lark }\end{array}$ & $\begin{array}{l}\text { Ammomanes } \\
\text { phoenicurus }\end{array}$ & LC & $\mathrm{R}$ & 1 & 2 \\
\hline $\begin{array}{l}\text { Malabar Crested } \\
\text { Lark }\end{array}$ & $\begin{array}{l}\text { Galerida } \\
\text { malabarica }\end{array}$ & LC & $\mathrm{R}$ & 1 & 2 \\
\hline Eastern Skylark & Alauda gulgula & LC & $R$ & I & 1 \\
\hline \multicolumn{6}{|l|}{ Hirundinidae } \\
\hline Dusky Crag-Martin & Hirundo concolor & LC & $\mathrm{R}$ & I & 4 \\
\hline House Swallow & Hirundo tahitica & LC & $\mathrm{R}$ & 1 & 4 \\
\hline $\begin{array}{l}\text { Red-rumped } \\
\text { Swallow }\end{array}$ & Hirundo daurica & LC & RM & 1 & 3 \\
\hline Common Swallow & Hirundo rustica & LC & RM & 1 & 2 \\
\hline $\begin{array}{l}\text { Wire-tailed } \\
\text { Swallow }\end{array}$ & Hirundo smithii & LC & $\mathrm{R}$ & 1 & 3 \\
\hline \multicolumn{6}{|l|}{ Motacillidae } \\
\hline Forest Wagtail & $\begin{array}{l}\text { Dendronanthus } \\
\text { indicus }\end{array}$ & LC & RM & 1 & $1,2,3$ \\
\hline Yellow Wagtail & Motacilla flava & LC & RM & 1 & 3 \\
\hline White Wagtail & Motacilla alba & LC & RM & $\mathrm{F}$ & 2 \\
\hline Grey Wagtail & Motacilla cinerea & LC & M & I & 2,3 \\
\hline Large Pied Wagtail & $\begin{array}{l}\text { Motacilla } \\
\text { maderaspatensis }\end{array}$ & LC & $\mathrm{R}$ & 1 & 3 \\
\hline Paddyfield Pipit & Anthus rufulus & LC & $\mathrm{R}$ & I & 3 \\
\hline Nilgiri Pipit & $\begin{array}{l}\text { Anthus nilghiriensis } \\
*\end{array}$ & VU & $\mathrm{R}$ & 1 & 3 \\
\hline \multicolumn{6}{|l|}{ Campephagidae } \\
\hline $\begin{array}{l}\text { Black-headed } \\
\text { Cuckoo-Shrike }\end{array}$ & $\begin{array}{l}\text { Coracina } \\
\text { melanoptera }\end{array}$ & LC & $\mathrm{RM}$ & 1 & 2,3 \\
\hline $\begin{array}{l}\text { Pied Flycatcher- } \\
\text { Shrike }\end{array}$ & Hemipus picatus & LC & $\mathrm{R}$ & 1 & 3 \\
\hline $\begin{array}{l}\text { Large Cuckoo- } \\
\text { Shrike }\end{array}$ & Coracina macei & LC & $\mathrm{R}$ & 1 & 3 \\
\hline Scarlet Minivet & $\begin{array}{l}\text { Pericrocotus } \\
\text { flammeus }\end{array}$ & LC & $\mathrm{R}$ & 1 & 2,3 \\
\hline Large Woodshrike & $\begin{array}{l}\text { Tephrodornis } \\
\text { gularis }\end{array}$ & LC & $\mathrm{R}$ & 1 & 4 \\
\hline $\begin{array}{l}\text { Common } \\
\text { Woodshrike }\end{array}$ & $\begin{array}{l}\text { Tephrodornis } \\
\text { pondicerianus }\end{array}$ & LC & $\mathrm{R}$ & I & 2,3 \\
\hline \multicolumn{6}{|l|}{ Pycnonotidae } \\
\hline Black Bulbul & $\begin{array}{l}\text { Hypsipetes } \\
\text { leucocephalus }\end{array}$ & LC & $\mathrm{R}$ & $\mathrm{F}$ & 3 \\
\hline $\begin{array}{l}\text { Black-crested } \\
\text { Bulbul }\end{array}$ & $\begin{array}{l}\text { Pycnonotus } \\
\text { melanicterus }\end{array}$ & LC & $\mathrm{R}$ & $\mathrm{F}$ & 2,3 \\
\hline $\begin{array}{l}\text { Red-whiskered } \\
\text { Bulbul }\end{array}$ & Pycnonotus jocosus & LC & $\mathrm{R}$ & $\mathrm{F}$ & 2,3 \\
\hline $\begin{array}{l}\text { White-browed } \\
\text { Bulbul }\end{array}$ & $\begin{array}{l}\text { Pycnonotus } \\
\text { luteolus }\end{array}$ & LC & $\mathrm{R}$ & $\mathrm{F}$ & 3 \\
\hline Red-vented Bulbul & Pycnonotus cafer & LC & $\mathrm{R}$ & $\mathrm{F}$ & 2,3 \\
\hline $\begin{array}{l}\text { Yellow-browed } \\
\text { Bulbul }\end{array}$ & Iole indica & LC & $R$ & $\mathrm{~F}$ & 2,3 \\
\hline $\begin{array}{l}\text { Grey-headed } \\
\text { Bulbul }\end{array}$ & $\begin{array}{l}\text { Pycnonotus } \\
\text { priocephalus * }\end{array}$ & NT & $R$ & $\mathrm{~F}$ & 2,3 \\
\hline $\begin{array}{l}\text { Yellow-throated } \\
\text { Bulbul }\end{array}$ & $\begin{array}{l}\text { Pycnonotus } \\
\text { xantholaemus }\end{array}$ & VU & $\mathrm{R}$ & $\mathrm{F}$ & 2 \\
\hline
\end{tabular}




\begin{tabular}{|c|c|c|c|c|c|}
\hline $\begin{array}{l}\text { Family / Common } \\
\text { Name }\end{array}$ & Scientific Name & IUCN & Status & Guild & Source \\
\hline \multicolumn{6}{|l|}{ Irenidae } \\
\hline Common lora & Aegithina tiphia & LC & $R$ & 1 & 2,3 \\
\hline $\begin{array}{l}\text { Asian Fairy- } \\
\text { Bluebird }\end{array}$ & Irena puella & LC & $\mathrm{R}$ & $\mathrm{F}$ & 2,3 \\
\hline Jerdon's Chloropsis & $\begin{array}{l}\text { Chloropsis } \\
\text { cochinchinensis }\end{array}$ & LC & $\mathrm{R}$ & 1 & 2,3 \\
\hline $\begin{array}{l}\text { Gold-fronted } \\
\text { Chloropsis }\end{array}$ & $\begin{array}{l}\text { Chloropsis } \\
\text { aurifrons }\end{array}$ & LC & $\mathrm{R}$ & 1 & 3 \\
\hline \multicolumn{6}{|l|}{ Laniidae } \\
\hline Brown Shrike & Lanius cristatus & LC & $M$ & 1 & 2,3 \\
\hline $\begin{array}{l}\text { Southern Grey } \\
\text { Shrike (?) }\end{array}$ & Lanius meridionalis & LC & RM & 1 & 3 \\
\hline $\begin{array}{l}\text { Rufous-backed } \\
\text { Shrike }\end{array}$ & Lanius schach & LC & $\mathrm{R}$ & 1 & 2,3 \\
\hline Bay-backed Shrike & Lanius vittatus & LC & $R$ & 1 & 2,3 \\
\hline \multicolumn{6}{|l|}{ Muscicapidae } \\
\hline $\begin{array}{l}\text { Blue-headed Rock- } \\
\text { Thrush }\end{array}$ & $\begin{array}{l}\text { Monticola } \\
\text { cinclorhynchus }\end{array}$ & LC & RM & 1 & 1,2 \\
\hline Blue Rock-Thrush & Monticola solitarius & LC & RM & 1 & 3 \\
\hline Indian Blue Robin & Luscinia brunnea & LC & RM & 1 & 1,3 \\
\hline Scaly Thrush & Zoothera dauma & LC & RM & 1 & 1 \\
\hline $\begin{array}{l}\text { Oriental Magpie- } \\
\text { Robin }\end{array}$ & Copsychus saularis & LC & $\mathrm{R}$ & 1 & 2,3 \\
\hline $\begin{array}{l}\text { Orange-headed } \\
\text { Thrush }\end{array}$ & Zoothera citrina & LC & $\mathrm{R}$ & 1 & 3 \\
\hline $\begin{array}{l}\text { White-rumped } \\
\text { Shama }\end{array}$ & $\begin{array}{l}\text { Copsychus } \\
\text { malabaricus }\end{array}$ & LC & $\mathrm{R}$ & I & 2,3 \\
\hline $\begin{array}{l}\text { Malabar Whistling- } \\
\text { Thrush (Image 2) }\end{array}$ & $\begin{array}{l}\text { Myiophonus } \\
\text { horsfieldii }\end{array}$ & LC & $\mathrm{R}$ & 1 & $1,2,3$ \\
\hline Indian Robin & $\begin{array}{l}\text { Saxicoloides } \\
\text { fulicata }\end{array}$ & LC & $\mathrm{R}$ & 1 & 2,3 \\
\hline Pied Bushchat & Saxicola caprata & LC & $R$ & 1 & 2,3 \\
\hline $\begin{array}{l}\text { White-bellied } \\
\text { Shortwing (Image } \\
\text { 3) }\end{array}$ & $\begin{array}{l}\text { Brachypteryx } \\
\text { major* }\end{array}$ & EN & $\mathrm{R}$ & 1 & 2,3 \\
\hline Spotted Babbler & Pellorneum ruficeps & LC & $R$ & 1 & 2,3 \\
\hline $\begin{array}{l}\text { Indian Scimitar- } \\
\text { Babbler }\end{array}$ & $\begin{array}{l}\text { Pomatorhinus } \\
\text { horsfieldii }\end{array}$ & LC & $R$ & 1 & 2,3 \\
\hline $\begin{array}{l}\text { Yellow-eyed } \\
\text { Babbler }\end{array}$ & $\begin{array}{l}\text { Chrysomma } \\
\text { sinense }\end{array}$ & LC & $\mathrm{R}$ & 1 & 3 \\
\hline Common Babbler & Turdoides caudatus & LC & $R$ & 1 & 1,2 \\
\hline $\begin{array}{l}\text { Rufous-bellied } \\
\text { Babbler }\end{array}$ & $\begin{array}{l}\text { Dumetia } \\
\text { hyperythra }\end{array}$ & LC & $R$ & 1 & 3 \\
\hline Jungle Babbler & Turdoides striatus & LC & $R$ & 1 & 2,3 \\
\hline $\begin{array}{l}\text { Black-headed } \\
\text { Babbler }\end{array}$ & $\begin{array}{l}\text { Rhopocichla } \\
\text { atriceps }\end{array}$ & LC & $R$ & 1 & 3 \\
\hline Large Grey Babbler & Turdoides malcolmi & LC & $R$ & 1 & 3 \\
\hline $\begin{array}{l}\text { White-headed } \\
\text { Babbler }\end{array}$ & Turdoides affinis & LC & $R$ & 1 & 3 \\
\hline Quaker Tit-Babbler & $\begin{array}{l}\text { Alcippe } \\
\text { poioicephala }\end{array}$ & LC & $\mathrm{R}$ & 1 & 1,3 \\
\hline Jungle Prinia & Prinia sy/vatica & LC & $R$ & 1 & 4 \\
\hline $\begin{array}{l}\text { Grey-breasted } \\
\text { Laughingthrush }\end{array}$ & Garrulax jerdoni * & NT & $R$ & 1 & 3 \\
\hline
\end{tabular}

\begin{tabular}{|c|c|c|c|c|c|}
\hline $\begin{array}{l}\text { Family / Common } \\
\text { Name }\end{array}$ & Scientific Name & IUCN & Status & Guild & Source \\
\hline $\begin{array}{l}\text { Indian Rufous } \\
\text { Babbler }\end{array}$ & $\begin{array}{l}\text { Turdoides subrufus } \\
*\end{array}$ & LC & $\mathrm{R}$ & 1 & 1,3 \\
\hline $\begin{array}{l}\text { Greenish Leaf- } \\
\text { Warbler }\end{array}$ & $\begin{array}{l}\text { Phylloscopus } \\
\text { trochiloides }\end{array}$ & LC & $M$ & 1 & 3 \\
\hline $\begin{array}{l}\text { Large-billed Leaf- } \\
\text { Warbler }\end{array}$ & $\begin{array}{l}\text { Phylloscopus } \\
\text { magnirostris }\end{array}$ & LC & M & 1 & 1,3 \\
\hline $\begin{array}{l}\text { Blyth's Reed- } \\
\text { Warbler }\end{array}$ & $\begin{array}{l}\text { Acrocephalus } \\
\text { dumetorum }\end{array}$ & LC & RM & 1 & 2,3 \\
\hline $\begin{array}{l}\text { Western Crowned } \\
\text { Warbler }\end{array}$ & $\begin{array}{l}\text { Phylloscopus } \\
\text { occipitalis }\end{array}$ & LC & RM & 1 & 1 \\
\hline Ashy Prinia & Prinia socialis & LC & $\mathrm{R}$ & 1 & 2,3 \\
\hline $\begin{array}{l}\text { Streaked Fantail- } \\
\text { Warbler }\end{array}$ & Cisticola juncidis & LC & $\mathrm{R}$ & 1 & 1 \\
\hline $\begin{array}{l}\text { Indian Great Reed- } \\
\text { Warbler }\end{array}$ & $\begin{array}{l}\text { Acrocephalus } \\
\text { stentoreus }\end{array}$ & LC & $\mathrm{R}$ & 1 & 3 \\
\hline Common Tailorbird & $\begin{array}{l}\text { Orthotomus } \\
\text { sutorius }\end{array}$ & LC & $\mathrm{R}$ & 1 & 2,3 \\
\hline $\begin{array}{l}\text { Broad-tailed Grass- } \\
\text { Warbler }\end{array}$ & $\begin{array}{l}\text { Schoenicola } \\
\text { platyura* }\end{array}$ & VU & $\mathrm{R}$ & 1 & 1,3 \\
\hline $\begin{array}{l}\text { Hume's Lesser } \\
\text { Whitethroat }\end{array}$ & Sylvia althaea & LC & $M$ & 1 & 4 \\
\hline Orphean Warbler & Sylvia hortensis & LC & M & I & 1 \\
\hline Verditer Flycatcher & Eumyias thalassina & LC & RM & । & 2,3 \\
\hline $\begin{array}{l}\text { Asian Brown } \\
\text { Flycatcher }\end{array}$ & $\begin{array}{l}\text { Muscicapa } \\
\text { dauurica }\end{array}$ & LC & RM & 1 & 2,3 \\
\hline $\begin{array}{l}\text { Brown-breasted } \\
\text { Flycatcher }\end{array}$ & Muscicapa muttui & LC & RM & 1 & 3 \\
\hline $\begin{array}{l}\text { Rusty-tailed } \\
\text { Flycatcher }\end{array}$ & $\begin{array}{l}\text { Muscicapa } \\
\text { ruficauda }\end{array}$ & LC & $M$ & I & 1 \\
\hline $\begin{array}{l}\text { Grey-headed } \\
\text { Flycatcher }\end{array}$ & $\begin{array}{l}\text { Culicicapa } \\
\text { ceylonensis }\end{array}$ & LC & $\mathrm{R}$ & 1 & 2,3 \\
\hline $\begin{array}{l}\text { Tickell's Blue- } \\
\text { Flycatcher }\end{array}$ & Cyornis tickelliae & LC & $\mathrm{R}$ & 1 & 2,3 \\
\hline $\begin{array}{l}\text { Black-and-Orange } \\
\text { Flycatcher (Image } \\
\text { 4) }\end{array}$ & $\begin{array}{l}\text { Ficedula nigrorufa } \\
*\end{array}$ & NT & $\mathrm{R}$ & 1 & 2,3 \\
\hline Nilgiri Flycatcher & $\begin{array}{l}\text { Eumyias } \\
\text { albicaudata* }\end{array}$ & NT & $\mathrm{R}$ & I & 3 \\
\hline $\begin{array}{l}\text { White-bellied } \\
\text { Blue-Flycatcher } \\
\text { (Image 5) }\end{array}$ & Cyornis pallipes * & LC & $\mathrm{R}$ & 1 & $1,2,3$ \\
\hline $\begin{array}{l}\text { Asian Paradise- } \\
\text { Flycatcher }\end{array}$ & $\begin{array}{l}\text { Terpsiphone } \\
\text { paradisi }\end{array}$ & LC & $\mathrm{RM}$ & I & 2,3 \\
\hline $\begin{array}{l}\text { Black-naped } \\
\text { Monarch- } \\
\text { Flycatcher }\end{array}$ & Hypothymis azurea & LC & $\mathrm{R}$ & 1 & 2,3 \\
\hline $\begin{array}{l}\text { White-browed } \\
\text { Fantail-Flycatcher }\end{array}$ & Rhipidura aureola & LC & $\mathrm{R}$ & 1 & 2 \\
\hline \multicolumn{6}{|l|}{ Paridae } \\
\hline $\begin{array}{l}\text { Black-lored Yellow } \\
\text { Tit }\end{array}$ & Parus xanthogenys & LC & $\mathrm{R}$ & 1 & 3 \\
\hline Great Tit & Parus major & LC & $\mathrm{R}$ & 1 & 2,3 \\
\hline \multicolumn{6}{|l|}{ Sittidae } \\
\hline $\begin{array}{l}\text { Velvet-fronted } \\
\text { Nuthatch }\end{array}$ & Sitta frontalis & LC & $\mathrm{R}$ & 1 & 2,3 \\
\hline Dicaeidae & & & & & \\
\hline
\end{tabular}




\begin{tabular}{|c|c|c|c|c|c|}
\hline $\begin{array}{l}\text { Family / Common } \\
\text { Name }\end{array}$ & Scientific Name & IUCN & Status & Guild & Source \\
\hline $\begin{array}{l}\text { Thick-billed } \\
\text { Flowerpecker }\end{array}$ & Dicaeum agile & LC & $\mathrm{R}$ & $\mathrm{N}$ & 3 \\
\hline Plain Flowerpecker & Dicaeum concolor & LC & $\mathrm{R}$ & $\mathrm{N}$ & 3 \\
\hline \multicolumn{6}{|l|}{ Nectariniidae } \\
\hline $\begin{array}{l}\text { Purple-rumped } \\
\text { Sunbird }\end{array}$ & $\begin{array}{l}\text { Nectarinia } \\
\text { zeylonica }\end{array}$ & LC & $\mathrm{R}$ & $\mathrm{N}$ & 2,3 \\
\hline Purple Sunbird & Nectarinia asiatica & LC & $\mathrm{R}$ & $\mathrm{N}$ & 3 \\
\hline Loten's Sunbird & Nectarinia lotenia & LC & $R$ & $\mathrm{~N}$ & 3 \\
\hline Little Spiderhunter & $\begin{array}{l}\text { Arachnothera } \\
\text { longirostra }\end{array}$ & LC & $\mathrm{R}$ & I & 2,3 \\
\hline Small Sunbird & $\begin{array}{l}\text { Nectarinia minima } \\
*\end{array}$ & LC & $\mathrm{R}$ & $\mathrm{N}$ & 2,3 \\
\hline \multicolumn{6}{|l|}{ Zosteropidae } \\
\hline Oriental White-eye & $\begin{array}{l}\text { Zosterops } \\
\text { palpebrosus }\end{array}$ & LC & $\mathrm{R}$ & I & 2,3 \\
\hline \multicolumn{6}{|l|}{ Fringillidae } \\
\hline Common Rosefinch & $\begin{array}{l}\text { Carpodacus } \\
\text { erythrinus }\end{array}$ & LC & RM & G & 3 \\
\hline \multicolumn{6}{|l|}{ Estrildidae } \\
\hline $\begin{array}{l}\text { White-throated } \\
\text { Munia }\end{array}$ & $\begin{array}{l}\text { Lonchura } \\
\text { malabarica }\end{array}$ & LC & $R$ & G & 2,3 \\
\hline $\begin{array}{l}\text { White-rumped } \\
\text { Munia }\end{array}$ & Lonchura striata & LC & $\mathrm{R}$ & G & 2,3 \\
\hline Spotted Munia & $\begin{array}{l}\text { Lonchura } \\
\text { punctulata }\end{array}$ & LC & $\mathrm{R}$ & G & 3 \\
\hline $\begin{array}{l}\text { Black-headed } \\
\text { Munia }\end{array}$ & Lonchura malacca & LC & $\mathrm{R}$ & G & 3 \\
\hline \multicolumn{6}{|l|}{ Passerinae } \\
\hline House Sparrow & Passer domesticus & LC & $\mathrm{R}$ & G & 2,3 \\
\hline \multicolumn{6}{|l|}{ Ploceinae } \\
\hline Baya Weaver & Ploceus philippinus & LC & $\mathrm{R}$ & G & 2,3 \\
\hline Sturnidae & & & & & \\
\hline
\end{tabular}

\begin{tabular}{|c|c|c|c|c|c|}
\hline $\begin{array}{l}\text { Family / Common } \\
\text { Name }\end{array}$ & Scientific Name & IUCN & Status & Guild & Source \\
\hline Jungle Myna & Acridotheres fuscus & LC & $\mathrm{R}$ & I & 2,3 \\
\hline Southern Hill-Myna & Gracula indica & LC & $\mathrm{R}$ & $\mathrm{F}$ & 2,3 \\
\hline Brahminy Starling & Sturnus pagodarum & LC & RM & $\mathrm{F}$ & $1,2,3$ \\
\hline Rosy Starling & Sturnus roseus & LC & M & $\mathrm{F}$ & 1,3 \\
\hline $\begin{array}{l}\text { Grey-headed } \\
\text { Starling }\end{array}$ & $\begin{array}{l}\text { Sturnus } \\
\text { malabaricus }\end{array}$ & LC & $\mathrm{R}$ & $\mathrm{F}$ & 1,3 \\
\hline Common Myna & Acridotheres tristis & LC & $\mathrm{R}$ & $\mathrm{O}$ & 2,3 \\
\hline \multicolumn{6}{|l|}{ Oriolidae } \\
\hline Black-naped Oriole & Oriolus chinensis & LC & RM & $\mathrm{F}$ & 3 \\
\hline $\begin{array}{l}\text { Eurasian Golden } \\
\text { Oriole }\end{array}$ & Oriolus oriolus & LC & RM & $\mathrm{F}$ & 2,3 \\
\hline $\begin{array}{l}\text { Black-headed } \\
\text { Oriole }\end{array}$ & Oriolus xanthornus & LC & $\mathrm{R}$ & $\mathrm{F}$ & 2,3 \\
\hline \multicolumn{6}{|l|}{ Dicruridae } \\
\hline Ashy Drongo & \begin{tabular}{|l} 
Dicrurus \\
leucophaeus
\end{tabular} & LC & RM & I & 3 \\
\hline Black Drongo & $\begin{array}{l}\text { Dicrurus } \\
\text { macrocercus }\end{array}$ & LC & $\mathrm{R}$ & 1 & 2,3 \\
\hline Bronzed Drongo & Dicrurus aeneus & LC & $\mathrm{R}$ & I & 3 \\
\hline $\begin{array}{l}\text { Greater Racket- } \\
\text { tailed Drongo }\end{array}$ & Dicrurus paradiseus & LC & $\mathrm{R}$ & I & 2,3 \\
\hline $\begin{array}{l}\text { White-bellied } \\
\text { Drongo }\end{array}$ & \begin{tabular}{|l} 
Dicrurus \\
caerulescens
\end{tabular} & LC & $\mathrm{R}$ & I & 2,3 \\
\hline \multicolumn{6}{|l|}{ Corvidae } \\
\hline Indian Treepie & $\begin{array}{l}\text { Dendrocitta } \\
\text { vagabunda }\end{array}$ & LC & $\mathrm{R}$ & 0 & 2,3 \\
\hline $\begin{array}{l}\text { White-bellied } \\
\text { Treepie (Image 6) }\end{array}$ & $\begin{array}{l}\text { Dendrocitta } \\
\text { leucogastra }\end{array}$ & LC & $\mathrm{R}$ & 0 & $1,2,3$ \\
\hline House Crow & Corvus splendens & LC & $\mathrm{R}$ & 0 & 2,3 \\
\hline Jungle Crow & $\begin{array}{l}\text { Corvus } \\
\text { macrorhynchos }\end{array}$ & LC & $\mathrm{R}$ & 0 & $1,2,3$ \\
\hline
\end{tabular}

Endemic birds are marked with asterisk.

IUCN Status: CR - Critically Endangered, EN - Endangered, VU - Vulnerable, NT - Near-threatened, LC - Least Concern

Status: R - Resident, RM - Resident Migratory, M - Migratory

Feeding guild: A - Aquatic vegetarians, G - Granivores, F - Frugivore, P - Piscivores, C - Carnivore (including carrion feeders), O - Omnivores, N - Nectarivores Source: 1 - Nichols (1944a,b; 1945), 2 - Bhupathy et al. (2009 \& 2012), 3 - Babu (pers. obser.), 4 - Rajah Jayapal (pers. comm.)

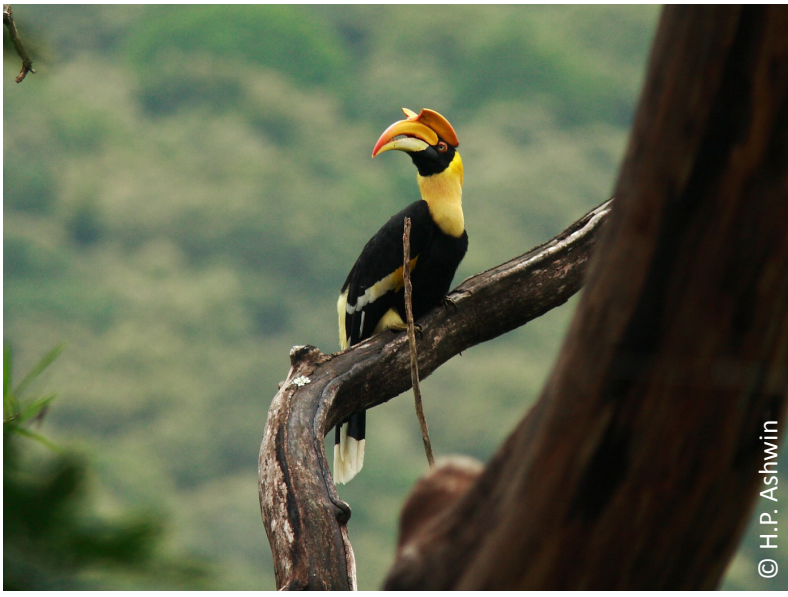

Image 1. Great Pied Hornbill

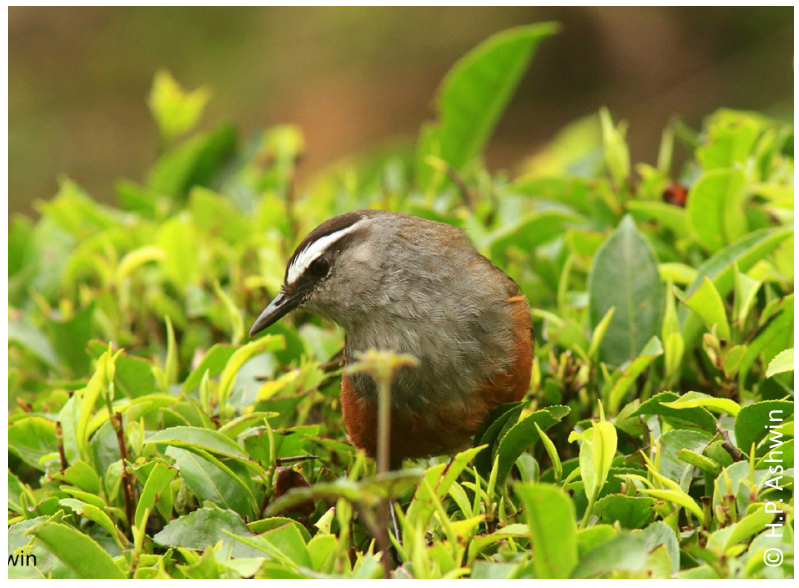

Image 2. Malabar Laughing-thrush 


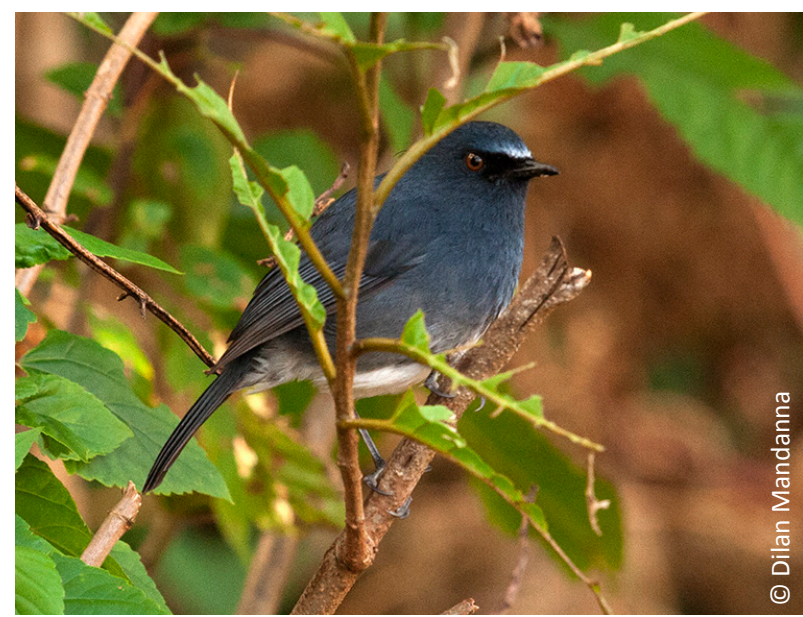

Image 3. White-bellied Shortwing

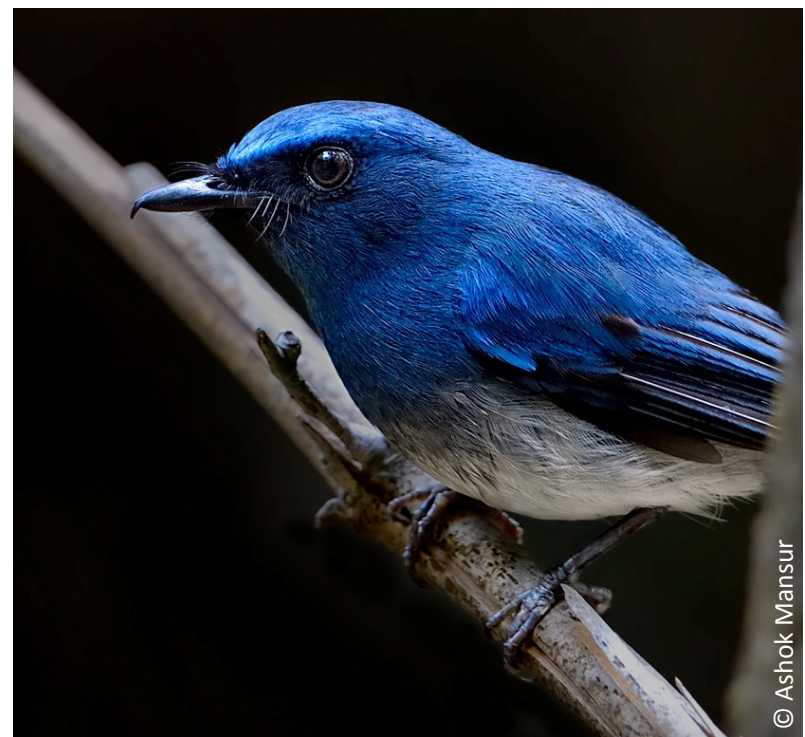

Image 5. White-bellied Blue Flycatcher

\section{REFERENCES}

Ali, S. \& D. Ripley (1983). Handbook of the birds of India and Pakistan Compact Edition, Oxford University Press, Mumbai, 737pp.

Balachandran, S., A.R. Rahmani, N. Ezhilarasi, S. Babu, J.P.P. Chakravarthy \& T. Ramesh (2005). Revaluation of bird community structure of Palni Hills with special reference to threatened and endemic species. Final Report. Bombay Natural History Society, Mumbai, 105pp.

Bhupathy, S., G. Srinivas \& N. Sathishkumar (2009). A Study on the Herpetofaunal Communities of the Upper Vaigai Plateau, Western Ghats, India. Final Report Submitted to the Ministry of Environment and Forests. Sálim Ali Centre for Ornithology and Natural History, Coimbatore, $75 \mathrm{pp}$.

Bhupathy, S., G. Srinivas., N. Sathishkumar, M. Murugesan, S. Babu, R. Suganthasakthivel \& P. Sivakumar (2012). Diversity and conservation of selected biota of the Meghamalai landscape, Western Ghats, India. Current Science 102(4): 590-595.

Bhupathy, S. \& S. Babu (2013). Meghamalai landscape : a biodiversity

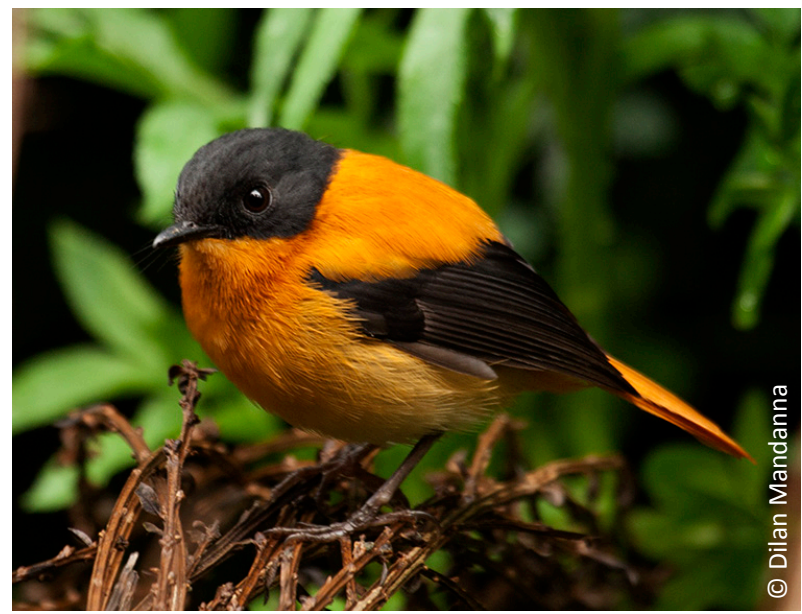

Image 4. Black-and-Orange Flycatcher

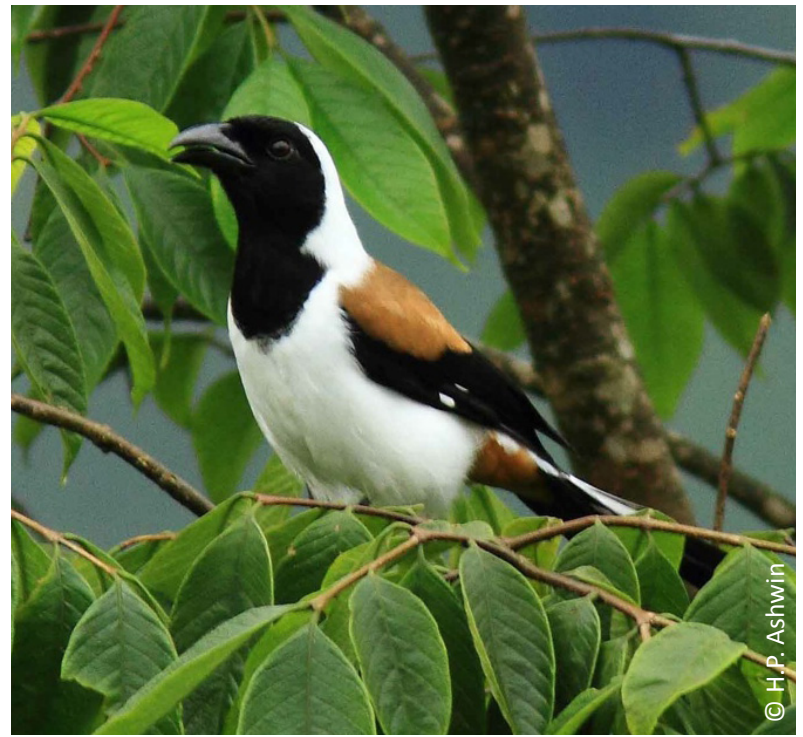

Image 6. White-bellied Treepie

hotspot. Journal of Threatened Taxa 5(15): 4939-4944; http:// dx.doi.org/10.11609/JoTT.o3592.4939-44

Biddulph, B.H. (1956). Occurrence of the Red-faced Malkoha (Phaenicophaeus pyrrhocephalus) Pennant in Madurai District: Madras presidency. Journal of the Bombay Natural History Society 53(1): 697-698.

CEPF (2007). Ecosystem profile: Western Ghats and Sri Lanka biodiversity hotspot, Western Ghats region, 95pp.

Daniels, R.J (2003). Biodiversity of the Western Ghats - an overview. In: Gupta, A.K., A. Kumar \& V. Ramakantha (eds.). ENVIS Bulletin: Wildlife \& Protected Areas, Conservation of Rainforests in India, 4(1): 229-242.

Gokula, V. \& L. Vijayan (1996). Birds of Mudumalai Wildlife Sanctuary, India. Forktail 12: 143-152.

Hoffmann, T.W. (1996). New bird records in Sri Lanka and some connected matters. Journal of the Bombay Natural History Society 96: 382-388.

IUCN (2012). IUCN Red List of Threatened Species. Version 2012.1. Electronic database accessible at http://www.iucnredlist.org/ 
captured on 25 July 2012.

Johnsingh, A.J.T (2001). The Kalakad-Mundanthurai Tiger Reserve: A global heritage of biological diversity. Current Science 80(3): 378388.

Johnsingh, A.J.T. \& J. Joshua (1994). Avifauna in three vegetation types on Mundanthurai Plateau, south India. Journal of Tropical Ecology 10(3): 323-335; http://dx.doi.org/10.1017/S0266467400007999

Kannan, R. (1998). Avifauna of the Anamalai Hills (Western Ghats) of southern India. Journal of the Bombay Natural History Society 95(2): 193-214.

Manakadan, R. \& A. Pittie (2001). Standardized common and scientific names of the birds of the Indian subcontinent. Buceros 6(1): 1-37.

Myers, N., B.A. Mittermeier, C.G. Mittermeier, G.A.B. da Fonesca \& J. Kent (2000). Biodiversity hotspots for conservation priorities. Nature 403: 853-858; http://dx.doi.org/10.1038/35002501

Nair, S.C. (1999). The southern Western Ghats. A biodiversity conservation plan. New Delhi, 92pp.

Nichols, E.D (1944a). Occurrence of birds in Madura District, Part I. Journal of the Bombay Natural History Society 44(3): 387-407.

Nichols, E.D (1944b). Occurrence of birds in Madura District, Part II. Journal of the Bombay Natural History Society 44(4): 574-584.

Nichols, E.D (1945). Occurrence of birds in Madura District, Part III. Journal of the Bombay Natural History Society 45(2): 122-132.

Nirmala, T. (2002). Ecology of bird communities in the Anaikatty Hills,
Coimbatore. PhD Thesis, Bharathiar University, Coimbatore, 274pp.

Prakash, V., M.C. Bishwakarma, A. Chaudhary, R. Cuthbert, R. Dav, M. Kulkarni, S. Kumar, K. Paudel, S. Ranade, R. Shringarpure \& R.E. Green (2012). The Population Decline of Gyps Vultures in India and Nepal Has Slowed since Veterinary Use of Diclofenac was Banned. PLoS ONE 7(11): e49118. http://dx.doi.org/10.1371/journal. pone. 0049118

Recher, H.F. \& W.E. Davis (2002). Foraging profile of a Salmon Gum woodland avifauna in Western Australia. Journal of Royal Society of Western Australia 85: 103-111.

Islam, M.Z. \& A.R. Rahmani (2004). Important Bird Areas: Priority sites for conservation. Indian Bird Conservation Network, Mumbai, $1133 p p$.

Rasmussen, P.C. \& J.C. Anderton (2005). Birds of South Asia. The Ripley Guide. Smithsonian Institution and Lynx Editions, 1072pp.

Somasundaram, S. \& L. Vijayan (2008). Foraging behavior and guild structure of birds in the Montane wet temperate forests of the Palni Hills, south India. Podoces 3(1/2): 79-91.

Statersfield, A.J., M.J. Crosby., A.J. Long \& D.C. Wege (1998). Endemic Bird Areas of The world: Priorities for Biodiversity Conservation. Birdlife International, Cambridge, 846pp.

Tamil Nadu Government Gazette (2009). Declaration of Meghamala Wildlife Sanctuary. Regd. No. TN/CCN/467/2009-11: 322-325. 